Exp Brain Res (1984) 56: 79-91

\title{
Effects of damage to superior colliculi and pre-tectum on movement discrimination in rhesus monkeys
}

\author{
A. Cowey, B. Smith, and C.M. Butter* \\ Department of Experimental Psychology, South Parks Road, Oxford, OXI 3UD, England
}

\begin{abstract}
Summary. Eight rhesus monkeys (Macaca mulatta) were trained to detect an instantaneous lateral displacement of a small spot of light. The smallest movement for $79 \%$ correct performance was then determined. Severing the splenium of the corpus callosum, which has to be done to reveal the superior colliculi, had no effect on the movement threshold. But when the superior colliculi were damaged in addition, there was an initial substantial impairment in the detection of movement and a smaller but permanent elevation in the threshold. Only if the rostral superior colliculi and pretectum were spared, was there no change in threshold, and there was evidence that the pretectal rather than collicular damage may be more important in relation to movement discrimination. Tests with human observers performing the same task showed that the threshold can be elevated by imprecise fixation and accomodation, suggesting that the consequences of mid-brain damage on movement detection may be wholly or in large part attributed to oculomotor disorders.
\end{abstract}

Key words: Superior colliculus - Pretectum - Visual movement - Oculomotor disorders - Monkeys

\section{Introduction}

In an earlier experiment on the ability of rhesus monkeys to discriminate between real and apparent visual movement (Collin and Cowey 1980) we discovered that ablation of the superior colliculi significantly increased the threshold distance over which the animals could just detect an instantaneous real displacement of a small spot of light in total darkness. We related the result to electrophysiological experi-

\footnotetext{
* Present address: Neuroscience Laboratory and Dept. of Psychology, Univ. of Michigan, Ann Arbor, USA.

Offprint requests to: A. Cowey (address see above)
}

ments which showed that many cells in the superior colliculi of monkeys are especially sensitive to moving stimuli, including discontinous jerky movements (Horn and Hill 1966; Humphrey 1968; Schiller and Koerner 1971; Cynader and Berman 1972; Goldberg and Wurtz 1972; Marrocco and Li 1977; Robinson and Wurtz 1976). In that experiment two spots, approximately $20^{\circ}$ apart, were presented and the animal had to decide which spot moved. It is therefore possible that the impairment we reported reflects the peripheral neglect that follows collicular damage (Denny-Brown 1962; Latto and Cowey 1971; Milner et al. 1978; Albano et al. 1982), rather than an impairment of movement discrimination per se. For example, if the spot which moves on any particular trial is not the spot which the animal is fixating the movement may be undetected because it occurs in a peripheral region of visual neglect and not because the threshold is normally higher for eccentric targets, or not only for this reason. The net result would be an overall increase in threshold, unless the normal animal always responds by examining one spot and deciding whether it moved or not, in which case the other spot would then be irrelevant on that trial.

In order to eliminate the possible contribution of a peripheral visual neglect we therefore repeated our previous experiment but presented only one stimulus spot, and trained animals to detect whether it had moved or not. When only a single stimulus is presented there is no uncertainty about where to look and behaviour should be solely governed by the ability of the animal to detect movement in the centre of the visual field. If the superior colliculus plays a special role in movement detection, as often suggested in connexion with the electrophysiological results cited above, monkeys with collicular ablation should still be impaired under this condition. However, it is possible that collicular damage impairs the ability of an animal to fixate promptly on a target 
which may move shortly after it appears. We therefore tested human observers on the same movement task, but with varying degrees of eccentric fixation to determine whether fixating eccentrically simulated any effects of collicular damage in monkeys.

\section{Methods}

\section{Subjects}

Eight juvenile rhesus monkeys (Macaca mulatta) were used; three were females (Nos. 1,4 and 5) and the remaining five were males. All were experimentally naive at the start of the investigation. They were tested each day when moderately hungry. All gained weight throughout the experiment.

\section{Apparatus - movement thresholds}

The animal was tested within a transport cage which could be locked in place within the apparatus. The back of the cage was a clear 'Perspex' panel through which the animal could be watched on a video monitor. The front of the cage had widely spaced horizontal bars between which the animal could see and reach a visual display located $20 \mathrm{~cm}$ beyond the bars. The display consisted of two clear 'Perspex' response panels $10 \mathrm{~cm}$ in diameter and connected to micro-switches. Immediately behind the right-hand panel was a 'Telequipment' D34 oscilloscope screen with P31 phosphor, and the $\mathrm{Y}$ axis mounted horizontally. Just below and between the two panels was a $3 \mathrm{~cm}$ diameter clear 'Perspex' button which was illuminated between trials. Below this was a foodwell into which a peanut could be automatically delivered. Between trials the transport cage was illuminated from above with a $15 \mathrm{w}$ house light. This visible light was extinguished during trials and replaced with an infra-red light which was presumably invisible to the animal but provided a clear picture on the video monitor. Apart from the light from the stimulus, the animal was therefore in darkness during each trial. Thus, if the animals' perception is similar to ours, there was no visible frame of reference against which the animal might infer movement from a change in position of the stimulus.

The stimulus was a spot of light, of diameter $0.5 \mathrm{~mm}$ and luminance $8.5 \mathrm{~cd} \cdot \mathrm{m}^{-2}$, centred on the face of the oscilloscope behind the right-hand response panel. Luminance was measured with a digital photometer (Gamma Scientific, Model 2400) calibrated against a radioactive beta light of known luminance. By a step-change in the input voltage to the $\mathrm{Y}$-axis amplifier the spot could be moved horizontally to the monkey's right. The movement always occurred over a period of $0.6 \mathrm{~ms}$, and perceptually the spot appeared to human observers to jump from one point to another. The apparatus was automatically controlled by logic modules in an adjacent room where the animal's performance was álso recorded.

\section{Procedure - movement thresholds}

The monkey started each trial by pressing the illuminated button. This immediately extinguished the button-light and house-light, turned on the infra-red light, and presented a spot of light on the right-hand screen. After a fixed delay of $2 \mathrm{~s}$, the spot either moved or remained stationary according to a random schedule. The correct response was to press the right-hand panel if the spot moved and the left-hand one if it remained still. If the animal responded correctly, a peanut was delivered to the food-well and the house-light came on. If the animal pressed the wrong panel it was not rewarded, a warbling tone was presented for $3 \mathrm{~s}$ via an overhead loudspeaker, and the house-light remained extinguished for $5 \mathrm{~s}$ before the start of the next intertrial interval. On the rare occasions when the animal responded before $2 \mathrm{~s}$ had elapsed or failed to respond within $5 \mathrm{~s}$ the trial was ended and discounted. Masking white noise was present throughout testing.

The animals first learned to press the illuminated button to turn on the spot, which then allowed them to press either panel to obtain a peanut. In the next stage the spot either oscillated horizontally at $2-5 \mathrm{~Hz}$ for $10 \mathrm{~s}$ over a distance of $3 \mathrm{~cm}$, in which case the correct response was to press the panel in front of the spot, or it remained stationary, in which case the animal was rewarded for pressing the left panel. Once this discrimination had been learned the duration of the oscillation was progressively reduced until only one $3 \mathrm{~cm}$ displacement occurred and the animal was correctly discriminating between a single movement and no movement on more than $90 \%$ of the trials. Once this task had been mastered (a mean of 9580 trials) the distance over which the spot moved was systematically reduced and thresholds were measured using a titration procedure. The distance moved by the spot was reduced by $10 \%$ whenever the animal responded correctly on three consecutive trials on which the spot moved. Whenever the animal responded incorrectly on a movement trial the displacement was increased by $10 \%$. The animal's performance on the nomovement trials, on which errors were very rare, had no effect on the titration. This procedure ensured that the animal's performance stabilised at about $80 \%$ correct (Wetherill and Levitt 1965). Each session started with the final value of the distance over which the spot moved in the preceding session. The mean of the changeover points at which the spot displacement was changed from a reduction to an increase, or vice versa in each session, was calculated to give the mean displacement threshold for each session.

Titration testing was continued until a monkey's performance had stabilised at a level beyond which no further improvement was expected. This stability was defined by two criteria. To satisfy the first, the mean of the daily thresholds calculated from the last block of 15 sessions had to be equal to or greater than the mean threshold for the immediately preceding block of 15 sessions. This ensured that there had been no improvement over 30 sessions. To fulfil the second criterion the standard error of the mean threshold in these 30 sessions had to be less than $5 \%$. When both criteria had been satisfied the final overall threshold was expressed as the mean of the 30 sessions. The mean number of trials to reach criterion was 5140. Post-operatively, i.e. after removal of the superior colliculi (experimental group) or section of the splenium of the corpus callosum (control group), threshold testing began about two weeks after operation. Throughout all the experiments daily sessions consisted of 100 trials, randomly presented as movement or no-movement trials, with the proviso that from 48-52 trials of each kind had to occur.

\section{Procedure - intensity discrimination thresholds}

After post-operative movement thresholds had been measured and various control procedures completed (see below), the ability of the animals to detect a step increment in luminance was then measured, using the same apparatus, titration procedure, and criterion for threshold. As this was a new task which had not been presented before operation, the pre- and post-operative performance could not be compared in individual animals, except in the 3 operated controls, in which the superior colliculi were subsequently ablated. On each trial, the spot appeared as before but the animal's task was to decide whether there was an increase in its 
luminance after $2 \mathrm{~s}$. The initial luminance could be varied but on trials on which it increased the final luminance was always $100 \mathrm{~cd} . \mathrm{m}^{-2}$. If there was an increase in luminance the animal had to press the panel in front of the spot to receive reward. If the spot did not change in intensity the left panel had to be pressed to receive reward. The luminance was varied by a step change in voltage to the $\mathrm{Z}$-axis amplifier of the oscilloscope. For titration the luminance change was varied in steps of $1.75 \mathrm{~cd} . \mathrm{m}^{-2}$.

All the animals were tested on this new task once postoperative criterion had been reached on the movement experiment, and the three operated controls were re-tested about two weeks after their second operation. All animals transferred well from the movement discrimination to the intensity discrimination, requiring only a mean of 390 trials to learn the basic task to criterion of $90 \%$ correct, and a mean of 2050 trials to reach the beginning of the criterion for threshold. As in the previous experiment the animals were given 100 trials per session, randomly presented.

\section{Control procedures}

(a) Monkeys. Once all the animals had reached post-operative criterion on the movement experiment they were presented with two control tasks, in which the apparatus and testing procedure remained largely unaltered. In the first, the spot was maximally defocussed in an attempt to mimic the optical effects of temporarily paralysing accomodation. Each animal was given 5 consecutive testing sessions of 100 trials. In the second control procedure, one drop of either $0.25 \% \mathrm{w} / \mathrm{v}$ hyoscine hydrobromide or $0.9 \%$ saline, in both instances at body temperature, were put in the eyes, and after a delay of about half an hour the animal was tested on the normal movement discrimination. Each animal was restrained whilst the eye drops were applied in order to ensure that the animal did not immediately blink them out. The animals were given four days testing after each application of hyoscine or of saline. This procedure was carried out three times with hyoscine and twice with saline, starting with hyoscine and alternating thereafter. This procedure was repeated for the three operated controls, once stability had been reached. However, on the second set the order was reversed so that the series began with saline. Only one treatment was given each week, so that there was a 3 day interval between the end of one treatment and the beginning of the next.

(b) Human subjects. Running concurrently with each visual discrimination were control experiments with human observers which attempted to mimic and evaluate the strategies the monkeys may have been adopting. The same titration procedure was used. For the movement discrimination two of the authors (AC and BS) were subjects. The subject sat on a stool within the testing box, and the eyes were level with and $28.5 \mathrm{~cm}$ away from the stimulus panel unless otherwise stated. This distance was chosen (a) because $1 \mathrm{~cm}$ subtends exactly $2.0^{\circ}$ and (b) it was close to the distance between the monkeys and the stimulus. The following conditions were used:

(A) Stimulus spot directly in front of right eye, but both eyes fixating it

(B) Spot in front of right eye but subject looked at infinity, resulting in diplopia with a blurred image on the fovea of the right eye and on the parafovea of the left eye. This approximates the least disruptive conditions for a viewer looking at infinity

(C and D) Conditions $A$ and $B$ were repeated but at a viewing distance of $57 \mathrm{~cm}$. As the interpupillary distance in man is almost exactly twice that in rhesus monkeys these conditions ensure that the angular retinal disparity of double images will be the same as in a monkey viewing at $28.5 \mathrm{~cm}$.
(E) The subject was encouraged to move the head laterally between trials so that the stimulus was not necessarily directly in front of the right eye. The subject fixated the stimulus with both eyes when it appeared. This is the normal viewing condition of a monkey.

(F) Procedure as in E but the subject looked at infinity. This meant that the stimulus rarely fell on the fovea of either eye. This viewing condition should most closely resemble that of a monkey with faulty vergence and accomodation.

(G) The stimulus was fixated monocularly, the other eye being covered with a patch.

(H) Procedures as in $\mathrm{G}$, but in addition the viewing eye was accommodated for infinity.

(I) The subject performed the task while fixating binocularly on the stimulus or on a second, dimmer, spot at an eccentricity of $2,4,6$ and $8^{\circ}$ to left or right of the target. This condition should resemble that for a monkey that can converge and accommodate but whose fixation is imprecise.

In each control condition there were 400 trials, including 400 at each eccentricity in condition I.

Controls were also carried out on the intensity discrimination. There were 3 subjects, A.C., B.B. and N.B., all experienced observers in this apparatus. The viewing distance was again $28.5 \mathrm{~cm}$. Daily for 4 days the subject performed 500 trials, 100 each while fixating the spot or $1,2,3$, and $4^{\circ}$ to the right of it. These were not randomly presented but occurred in ascending or descending runs, two rums of each. In this way each subject completed 2000 trials. Human observers proved to be so sensitive on the intensity discrimination that stepsizes smaller than $1.75 \mathrm{~cd} . \mathrm{m}^{-2}$ had to be used in some conditions.

\section{Surgery}

All operations were performed under an operating microscope using sterile procedures. In 5 of the animals (nos. 4, 5, 6, 7, and 8) the splenium of the corpus callosum was sectioned to reveal the superior colliculi, which were removed. In the 3 remaining animals (nos. 1, 2, and 3) only the splenium was sectioned at the first operation and these animals initially served as operated controls. The superior colliculi were then removed at a second operation about 18 months later.

The animal was sedated with ketamine hydrochloride ("Vetalar' $10 \mathrm{mg} / \mathrm{kg}$, i.m.) then deeply anaesthetised with sodium thiopentone, i.v. ('Pentothal', May and Baker) for the duration of the operation. After placing the animal in a head-holder, a midline incision was made in skin and fascia. A rectangular flap of bone, $5 \mathrm{~cm}$ long and $3 \mathrm{~cm}$ wide, was removed from the mid-line and stored in chilled saline. The dura was cut and reflected along one side of the sagittal sinus and the splenium of the corpus callosum severed over a length of about $10 \mathrm{~mm}$ with a small-gauged sucker while gently separating the medial cortex from the falx. This was the extent of the lesion for the 3 control animals. In the other animals the meninges above the mid-brain were then cut and parted to reveal the superior colliculi, which were often covered anteriorly by the pineall gland. The habenular and posterior commissures formed a prominent landmark at the rostral border of the colliculi. An attempt was made to remove the whole of the rostral superior colliculus, corresponding to central vision. The lesion therefore involved the dorsal pre-tectum where the superior colliculus dips beneath it. The lesion was extended, laterally and caudally as far as possible but the borders of the colliculi were never visible. An attempt was also made not to disturb the superficial blood vessels near the rostral border which supply parts of the dorsomedial thalamus. There was no more than slight and brief bleeding in any animal. When this had ceased the dura was sewn and the bone flap replaced and secured at its corners. Fascia 

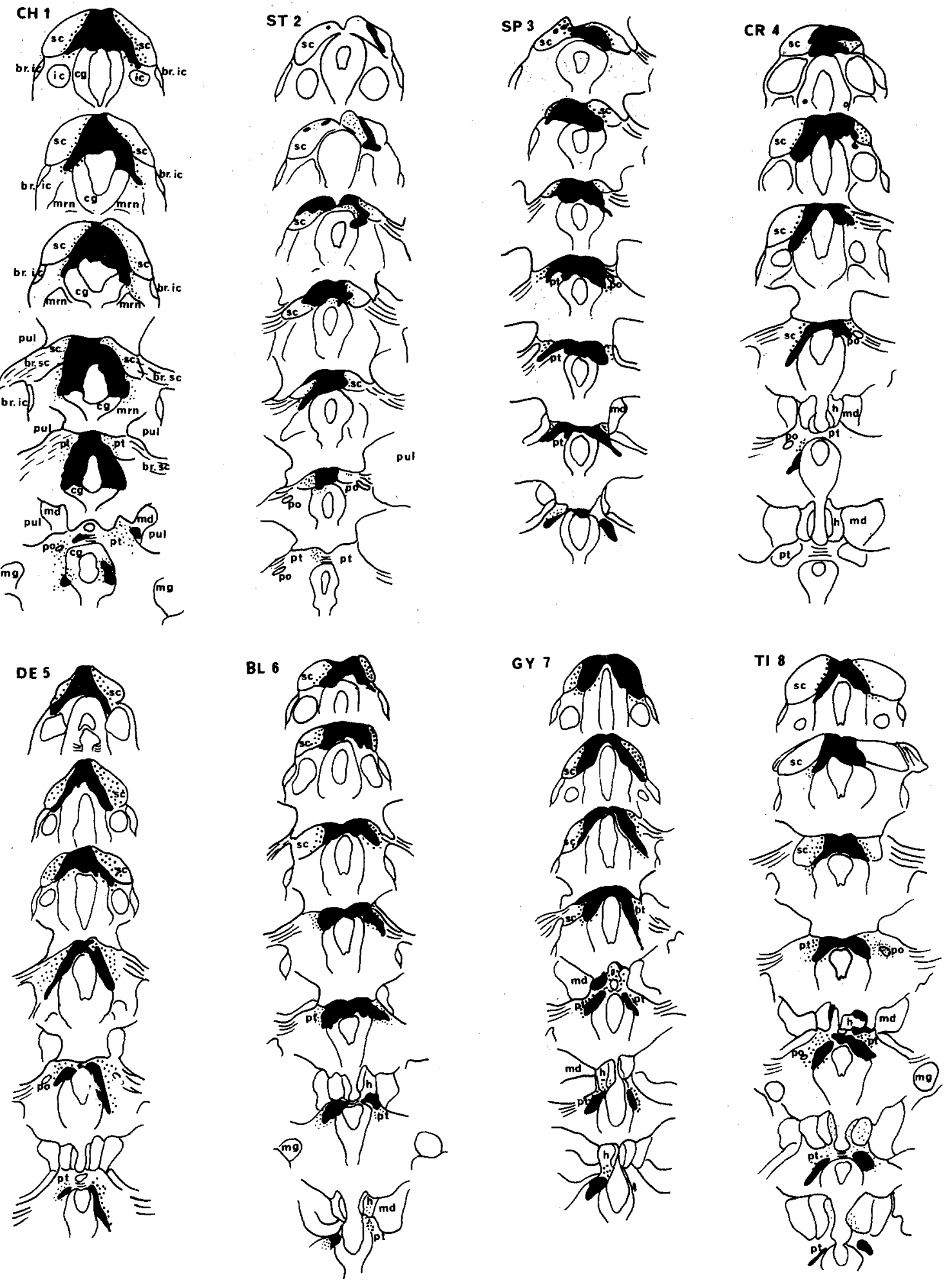

Fig. 1. Outline drawings of $50 \mu \mathrm{m}$ frontal sections, $0.5 \mathrm{~mm}$ apart, through the mid-brain from caudal (top) to rostral (bottom). Missing tissue is shown in black. Stippling indicates prominent gliosis. The most rostral extent of the lesion is shown on the bottom section for each animal. For clarity the most caudal tip of the lesion is not always shown. It was always within $0.5 \mathrm{~mm}$ of the uppermost section for each animal. The letters before each animal's number are a laboratory code to distinguish them from similarly numbered animals in other publications. To avoid clutter, all the relevant features were labelled only in the first animal. Abbreviations: sc, superior colliculus; ic, inferior colliculus; br, brachium; cg, central grey; mrn, mesencephalic reticular nucleus; pul, pulvinar nucleus; pt, pretectum; md, medialis dorsalis; po, olivary pretectal nucleus; $\mathrm{mg}$, medial geniculate nucleus; $\mathrm{h}$, habenular nucleus 
Table 1. The number of trials required to learn to detect a single movement of a luminous spot, the number of titration times then required to measure the smallest detectable movement, and the extent of the threshold in mon for 8 animals pre- and post-operatively. C-SC, refers to control operation in which the splenium of the corpus callosum was severed, followed by subsequent removal of the superior colliculi. SC refers to splenial transection and removal of superior colliculi in a single operation

\begin{tabular}{|c|c|c|c|c|c|c|c|c|c|}
\hline & & PRE-OP & & & POST-OP 1 & & & POST-OP & \\
\hline & $\begin{array}{l}\text { Trials } \\
\text { before } \\
\text { titration }\end{array}$ & $\begin{array}{l}\text { Titration } \\
\text { trials to } \\
\text { reach } \\
\text { criterion }\end{array}$ & $\begin{array}{l}\text { Threshold } \\
\text { displacement, } \\
\mathrm{mm}\end{array}$ & $\begin{array}{l}\text { Retraining } \\
\text { trials }\end{array}$ & $\begin{array}{l}\text { Titration } \\
\text { trials to } \\
\text { reach } \\
\text { criterion }\end{array}$ & $\begin{array}{l}\text { Threshold } \\
\text { displacement, } \\
\text { mm }\end{array}$ & $\begin{array}{l}\text { Retraining } \\
\text { trials }\end{array}$ & $\begin{array}{l}\text { Titration } \\
\text { trials to } \\
\text { reach } \\
\text { criterion }\end{array}$ & $\begin{array}{l}\text { Threshold } \\
\text { displacement, } \\
\mathrm{mm}\end{array}$ \\
\hline $1(\mathrm{C}-\mathrm{SC})$ & 7800 & 5400 & 0.36 & - & 600 & 0.35 & - & 2900 & 0.43 \\
\hline $2(\mathrm{C}-\mathrm{SC})$ & 6900 & 2800 & 0.38 & - & 400 & 0.24 & - & 100 & 0.23 \\
\hline $3(\mathrm{C}-\mathrm{SC})$ & 15900 & 300 & 4.00 & - & 5100 & 3.23 & 200 & 300 & 5.57 \\
\hline $4(\mathrm{SC})$ & 9700 & 3200 & 0.85 & - & 300 & 1.21 & - & - & - \\
\hline $5(\mathrm{SC})$ & 9500 & 6200 & 0.61 & - & 1600 & 1.51 & - & - & - \\
\hline $6(\mathrm{SC})$ & 7700 & 5900 & 0.46 & 1500 & 500 & 0.96 & - & - & - \\
\hline $7(\mathrm{SC})$ & 8000 & 5900 & 0.36 & 3100 & 5200 & 0.77 & - & - & - \\
\hline 8 (SC) & 11000 & 8700 & 1.66 & 3000 & 300 & 7.11 & - & - & - \\
\hline
\end{tabular}

and skin were then sewn. In the 3 control animals the superior colliculi were removed at a second operation by approaching them along the medial surface of the other hemisphere. Each animal was given 300,000 units of penicillin G ('Penidural L-A', Wyeth) at the end of the operation. All animals recovered promptly and there were no post-operative complications.

\section{Histology}

At the end of the experiment each animal was given a lethal intravenous dose of sodium pentobarbitone and perfused transcardially with $0.9 \%$ saline followed by $0.9 \%$ saline and $10 \%$ formalsaline. The dorsal skull was removed and the extent of the undamaged corpus callosum was measured while the head was in a stereotaxic frame. The brain was then trimmed in the stereotaxic frontal plane, and the block containing the mid-brain placed in $10 \%$ formalin and $30 \%$ sucrose until it sank. Frozen sections were cut at $50 \mu$ and every 5 th or 10 th section stained with cresyl violet. Where necessary additional sections were stained, either with cresyl violet or by Weil's method for myelin.

The results of the histology are shown in Fig. 1. In general the lesions were deep, involving all layers of the colliculus, and extended for the entire length of the colliculus at the mid-line. However the lateral edges of the colliculus were often spared, and the caudal pole in some instances. These regions of sparing are probably not important because (a) they were extensively undercut and (b) they are concerned with peripheral vision. Furthermore they were prominently spared in No. 8 , which was very impaired, but much less spared in No. 4 which was less impaired.

The more interesting histological findings concern the rostral colliculus and pre-tectum. Only in animal No. 2 were the most rostral colliculus and the pre-tectum spared bilaterally. As will be seen this animal was not impaired, or only briefly so. In animals 1 and 4 the posterior pre-tectum contained gliosis but there was little direct damage and the olivary nucleus could be seen on each side, although there was damage medial and ventral to it. The posterior commissure was normal in animals 1,2 and 4 except for the caudal edge in No. 1 . In the remaining animals $(3,5,6,7,8)$ there was direct and indirect damage to the posterior pretectum. This was most extensive in No. 8 , where the damage included the anterior pretectum, just in front of the posterior commissure, and parts of the habenular nuclei. In addition there was prominent demyelina- tion of the posterior commissure in animals $3,5,6,7,8$. There was no direct damage to the nuclei of Cajal, Darkschewitsch, or Bechterew, all of which have been implicated in the control of extrinsic or intrinsic eye muscles.

The rostro-caudal extent of the splenial section was estimated by measuring (a) the length of the remaining corpus callosum, which varied from $17-26 \mathrm{~mm}$, and (b) the distance from the posterior commissure to the posterior limit of intact corpus callosum, which varied from $0-4 \mathrm{~mm}$. The animals were separately ranked for (a) and (b), and a mean rank taken. From smallest to largest splenial section, the order was Nos. $5,7,3,8,1,4,6$, and 2 .

\section{Results}

\section{Preoperative learning}

Table 1 shows that every animal required several thousand trials $(6,900-15,900)$ before responding at better than $90 \%$ correct to a single $3 \mathrm{~cm}$ displacement of the spot. From watching the animals on the T.V. monitor it was clear that the protracted learning almost certainly reflected the slow improvement in the ability of each animal to attend to the screen so that it was always looking when the spot moved.

The second column of Table 1 shows that once the basic task had been mastered, fewer trials were required to reach the beginning of the 30 sessions $(3,000$ trials) over which performance was stable. The threshold was less than $0.5 \mathrm{~mm}$ in 4 animals but $4.0 \mathrm{~mm}$ in No. 3 , which showed little improvement once titration had begun and therefore reached the beginning of criterion within 300 trials. This animal rarely sat attentively in front of the display and frequently circled the cage even immediately after starting a trial. No animal was as good as two of the 
authors (AC, BS) whose binocular thresholds at a viewing distance of $28.5 \mathrm{~cm}$ and using the same testing procedure as for the monkeys were 0.17 and $0.19 \mathrm{~mm}$ (see Fig. 6).

\section{Neurological tests}

For the first two days after operation each animal was examined in the home cage with respect to the following: (1) Accuracy in reaching for peanuts and raisins held on the experimenter's palm; (2) orienting with eyes and head to small pieces of food presented to left, right, up and down with an eccentricity of at least $45^{\circ}$ in the visual field; (3) orientation to and tracking of small food objects moved in various directions in front of the animal; (4) spontaneous saccades; (5) strabismus; (6) spontaneous nystagmus; (7) retraction of the eyelids; (8) pupillary dilation, and pupillary contraction to a torchlight directed at the eyes; (9) blink reflex to a puff of air directed at the face and to a sudden hand movement directed at the face. If any unusual neurological signs were found the tests were repeated daily for one week. Any animal with persistent signs was occasionally examined over a period of months.

No unusual features were noted on any of the tests in the 3 control animals with section of the splenium of the corpus callosum. Following ablation of superior colliculus the most conspicuous effects, which occurred in all 8 animals, were a reduced number of spontaneous saccades, a neglect of objects presented peripherally, and poor visual tracking. It would be surprising if these were not related, but the neglect had usually disappeared within a week or two whereas the stare persisted for much longer. Animal No. 2 was least affected, and Nos. 7 and 8 most affected. In addition, intermittent misreaching and eyelid retraction were seen in Nos. $1,3,5,6,7$ and 8 . It was our impression that the pupils were incompletely dilated in Nos. 1, 3, 6, 7 and 8 and that this never fully recovered over a period of a year. However, pupillary constriction to bright light was always present, although it may not have been normal. The blink reflex was present and strabismus and nystagmus were not seen. This pattern of symptoms has been reported previously by several investigators (see Pasik et al. 1969a, b, for review).

It should be emphasised that more subtle neurological signs would almost certainly be impossible to detect by the methods we used. This means that the effects we detected were probably large, and that lessening effects probably outlasted our ability to detect them.
Table 2. The number of trials required to learn to detect an instantaneous increase in the intensity of a luminous spot, the number of titration trials then required to measure the smallest detectable increase, and the magnitude of the threshold as a percentage of the final intensity

\begin{tabular}{llllll}
\hline & \multicolumn{3}{c}{ POST-OP 1 } & \multicolumn{2}{c}{ POST-OP 2 } \\
\hline & $\begin{array}{l}\text { Trials } \\
\text { before } \\
\text { titration }\end{array}$ & $\begin{array}{l}\text { Titration } \\
\text { trials to } \\
\text { reach } \\
\text { criterion }\end{array}$ & $\begin{array}{l}\text { Threshold } \\
\text { luminance } \\
\text { change, } \%\end{array}$ & $\begin{array}{l}\text { Titration } \\
\text { trials to } \\
\text { reach } \\
\text { criterion }\end{array}$ & $\begin{array}{l}\text { Threshold } \\
\text { luminance } \\
\text { change, } \%\end{array}$ \\
\hline 1 (C-SC) & 600 & 200 & 24.6 & 9500 & 10.9 \\
2 (C-SC) & 200 & 2200 & 10.5 & 300 & 12.8 \\
3 (C-SC) & 200 & 6200 & 24.9 & 2600 & 20.4 \\
4 (SC) & 200 & 200 & 18.3 & - & - \\
5 (SC) & 200 & 200 & 17.7 & - & - \\
6 (SC) & 200 & 500 & 22.1 & - & - \\
7 (SC) & 200 & 3200 & 27.8 & - & - \\
8 (SC) & 1300 & 3700 & 31.3 & - & - \\
\hline
\end{tabular}

\section{Postoperative movement thresholds}

Table 1 shows that sectioning the splenium of the corpus callosum in animals 1,2 and 3 did not raise the threshold for detecting the movement of the spot. There was even a small improvement in two of the animals, presumably reflecting further extensive practice on the task. In contrast the threshold rose in the 5 animals with superior collicular ablation (Nos. 4-8) and the increase varied from $42 \%$ (No. 4) to $330 \%$ (No. 8). When first tested after operation, animals $6-8$ could not perform at better than $90 \%$ correct with a $3 \mathrm{~cm}$ movement and had to be retrained. Animals 7 and 8 had the largest lesions, involving rostral colliculus and posterior pretectum. The difference between the effects of splenial and splenial plus collicular ablation on the movement threshold was significant $\left(\mathrm{U}=0, \mathrm{~N}_{1}=3, \mathrm{~N}_{2}=5\right.$, $p=0.036,2$-tailed).

The results of subsequently removing the superior colliculus in the 3 control animals in which only the splenium was severed initially are shown to the right in Table 2. The movement threshold was raised in 2 animals but not in the third. In the latter case the mid-brain lesion was small and spared the whole of the rostral and lateral superior colliculus and the entire pretectum. The effect of the operations on the threshold for the detection of an instantaneous movement are shown for all 8 animals in Fig. 2, where the change in threshold is expressed as a ratio, i.e. the difference between preoperative and postoperative values divided by their sum. Using each animal as its own control the increase in threshold following tectal and pre-tectal damage was 


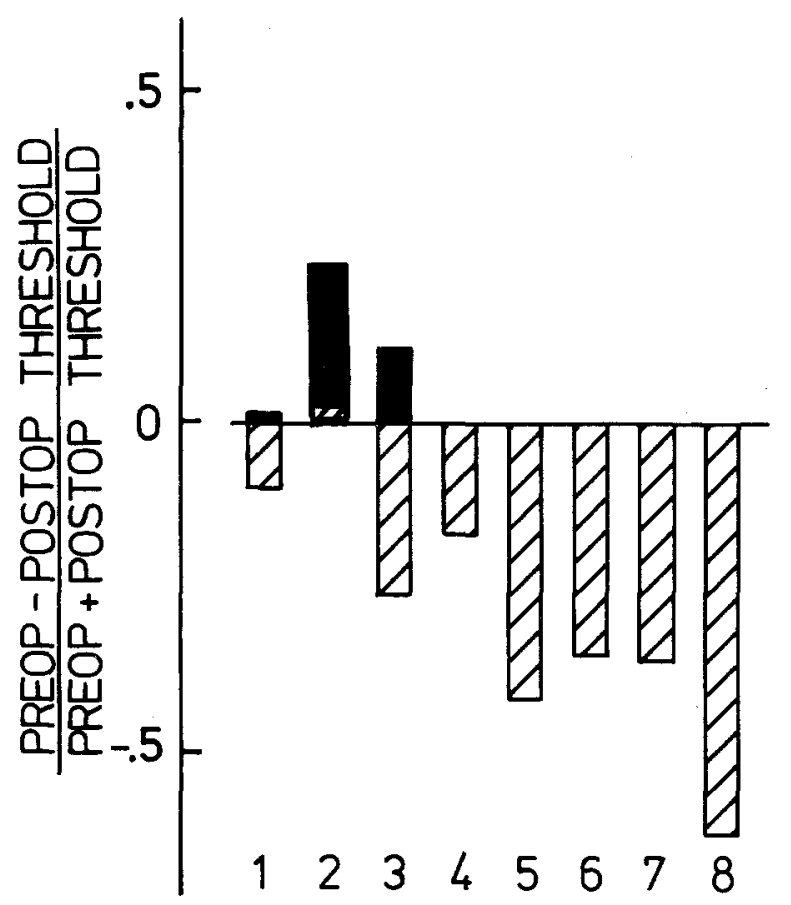

Fig. 2. Ratio of pre- and post-operative thresholds (difference divided by sum) for movement discrimination. Dark bars show effect of sectioning the splenium of the corpus callosum. Hatched bars show effects of mid-brain damage

significant $(\mathrm{N}=8, \mathrm{~T}=1, p<0.02$, 2-tailed, Wilcoxon Text).

Table 1 and Fig. 2 show only the final performance levels of the animals and give no indication of the severity of the movement discrimination impairment in the initial weeks after operation. The latter is apparent in Fig. 3, which plots performance over the first 3,500 titration trials after operation (i.e. after any necessary retraining trials) as a percentage of the pre-operative threshold, i.e. the mean of the last 3,000 trials before operation. In blocks 2 and 3 there was no overlap between the scores of the 3 surgical controls and the 8 collicular animals (which include the controls after their second operation). In the remaining blocks the single overlap was produced by animal No. 2, whose second operation spared the rostral colliculus and pretectum.

\section{Control procedures}

a. Spot defocus. If one effect of damaging the tectum and pretectum is to impair accommodation, such that the animal is unable to focus promptly or at all on close targets, and if this is a sufficient explanation for the impairment in discriminating movement, then it

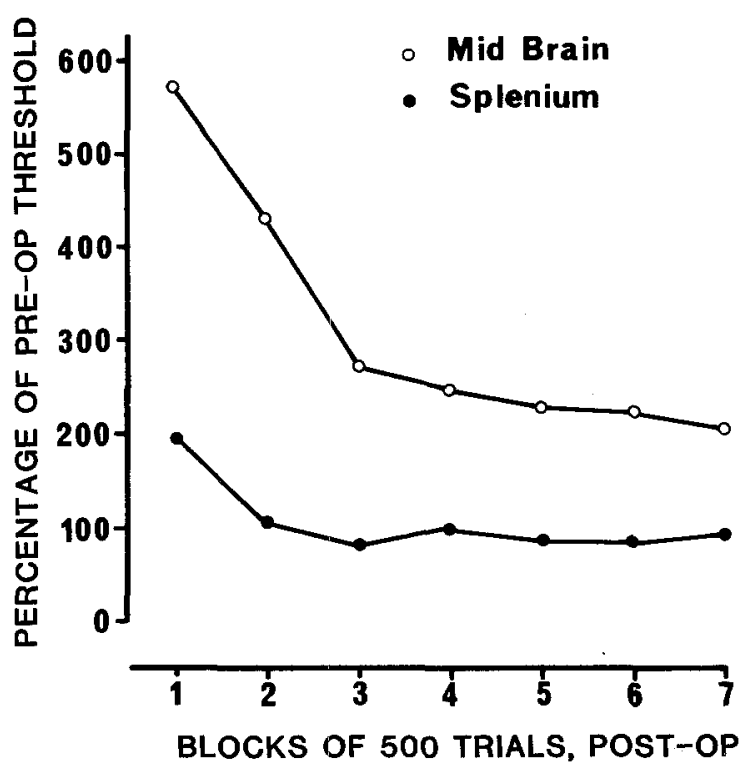

Fig. 3. Initial mean postoperative movement thresholds, expressed as a percentage of the final 3000 preoperative trials. Solid circles show effect of sectioning the splenium of the corpus callosum in Animals 1-3. Open circles show the effect of mid-brain damage in the same animals and in animals $4-8$

should be possible to mimic the effect of the lesion by deliberately defocussing the target. It was for this reason that all animals were tested after their first operation with the spot maximally defocussed by adjusting the focus and astigmatism controls on the oscilloscope. To a human observer the effect was to make the spot larger and fuzzy. As this procedure was carried out at the same time for all animals but at different times after each animal had reached postoperative criterion on the movement discrimination, the latter threshold values were not used for comparison. Instead each animal was given 500 trials (5 sessions) with the usual focussed spot followed immediately by 500 trials with the defocussed spot. The effects of defocussing are shown in Fig. 4. Defocussing the stimulus had less effect on the 3 animals with splenial section than the 5 animals with collicular ablation, but the group difference was statistically insignificant ( $\mathrm{U}=3, \mathrm{~N}_{1}=3, \mathrm{~N}_{2}=5, p=$ $0.11,2$-tailed). In other words, there was no evidence that the impairment of movement threshold in the collicular animals was solely caused by mild stimulus blurring for if it were the control animals should have been more affected than the collicular animals by defocussing the stimulus, and any impairment in the 3 control animals should have been comparable to that caused by mid-brain damage. All three control animals and three of the five collicular animals were barely affected by altering the stimulus itself. 


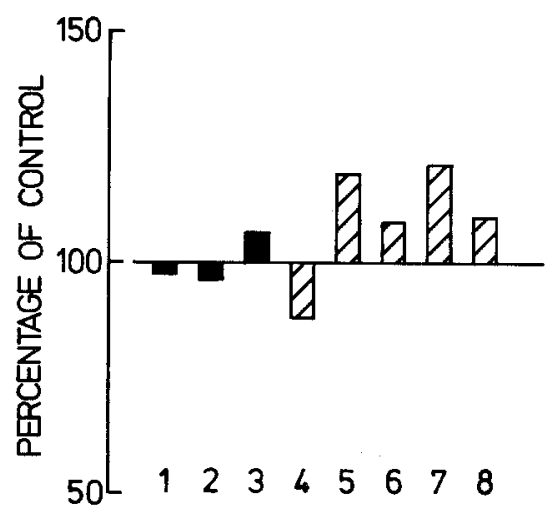

Fig. 4. Effects on movement threshold of defocussing the stimulus spot. The mean threshold over 500 consecutive times was compared with the preceding 500 control trials in which the spot was focussed on the screen. Black bars indicate splenial section; hatched bars indicate mid-brain damage

b. Relaxation of accomodation and the pupil. Although physically expanding and blurring the stimulus may resemble the effect on the retinal image of faulty accommodation, a better test is to keep the stimulus constant but relax accommodation. This was the reason for assessing the effect, after the first operation, of temporarily relaxing both accommodation and the pupil. In weeks 1,3 and 5 following attainment of the movement threshold criterion the cycloplegic agent was applied on day 1 , and the animal was tested daily for 4 days. On weeks 2 and 4 , the same procedure was used with one drop of $0.9 \%$ saline. All data from the first treatment were excluded from the analysis on the grounds that the animals may have been upset on the first occasion the drops were applied. To have included them would simply increase the effect to be described. Furthermore, only data from the first day of each treatment were used to compare hyoscine and saline because it was clear, as expected, that any effects had diminished by day 2 and disappeared by days 3 and 4 as the effects of the drug wore off. But it was necessary to demonstrate this recovery before the beginning of the subsequent treatment. Figure 5 therefore shows the ratio of performance for the first 200 trials with hysoscine and the first 200 trials with saline. The three surgical controls were more impaired than the five collicular animals by having their accommodation and pupils temporarily relaxed ( $\mathrm{U}=0, \mathrm{~N}_{1}=3, \mathrm{~N}_{2}=5, p=0.036,2$-tailed). This difference is not simply a consequence of hyoscine having its greatest effect on animals with low thresholds because Control No. 3, which had a high threshold (see Table 1) also showed the greatest effect. The result therefore supports the suggestion

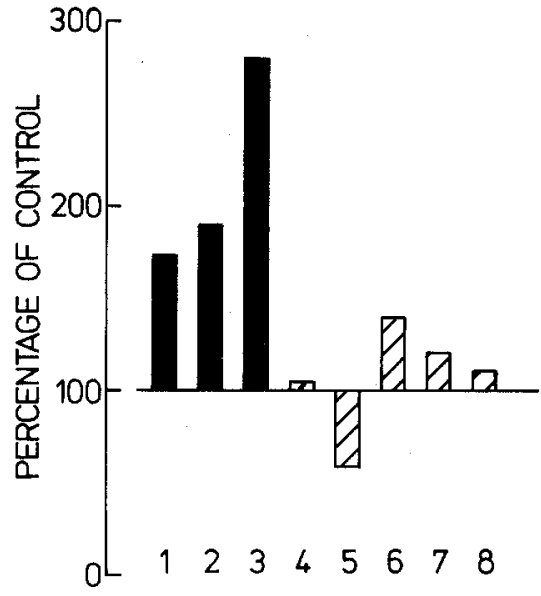

Fig. 5. Effects on movements threshold of temporarily relaxing accomodation and the pupil. Performance over 200 trials following administration of hyoscine hydrobromide is compared with performance over 200 control trials following administration of physiological saline. Black bars indicate animals with splenial section; hatched bars indicate animals with midbrain damage

that, at least in part, the elevation of the movement discrimination threshold after collicular damage is caused by image blurring as a result of pupillary expansion and faulty accommodation. However, this is probably at best a minor contribution because the approximately twofold acute elevation produced in control animals by hyoscine (see Fig. 5) is far smaller than that seen when the animals were first tested after a collicular operation (see Fig. 3). Indeed, the sixfold elevation seen initially after collicular ablation was even greater during the first days of testing, when 3 animals could not reliably perform with a $3 \mathrm{~cm}$ movement. Factors additional to image blurring must therefore be contributing to the effects of collicular ablation.

\section{Luminance increment thresholds}

Following the measurements of movement discrimination after the first operation, all 8 animals were tested in identical fashion on a task in which they had to detect an instantaneous brightening of the stimulus. All animals transferred promptly to this task, and 6 of them required only 200 trials to reach the criterion of $90 \%$ correct on the initial condition in which the intensity of the spot was increased from 60 to $100 \mathrm{~cd} \cdot \mathrm{m}^{-2}$. Table 2 shows that the number of titration trials then needed to reach the beginning of the 3,000 threshold trials varied from 200 to 6,200 , with no indication that the collicular group was impaired. As there were no pre-operative measures 


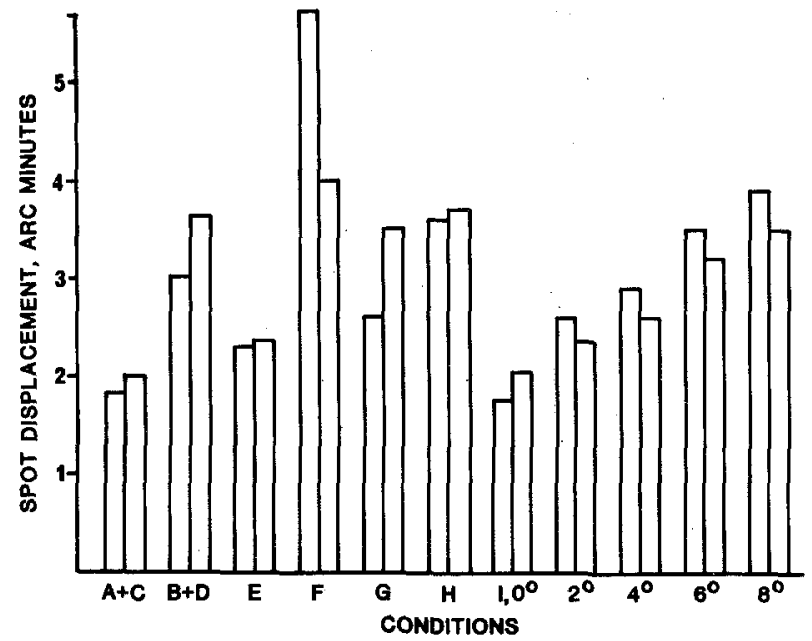

Fig. 6. Movement discrimination thresholds in are minutes for two human observers under various viewing conditions. For each observer there were 400 trials on each condition. A $+\mathrm{C}$, spot in front of right eye at 28.5 or $57 \mathrm{~cm}$ and fixated binocularly; $\mathrm{B}+\mathrm{D}$ spot in front of right eye at 28.5 or $57 \mathrm{~cm}$, observer looking at infinity; $\mathrm{E}$, as $\mathrm{A}+\mathrm{C}$ but head moved between trials; $\mathrm{F}$, as $\mathrm{E}$ but observer looking at infinity. Image of stimulus is blurred and rarely on either fovea; $\mathrm{G}$, monocular fixation of stimulus; $\mathrm{H}$, monocular fixation on infinity with blurred image on fovea; $I, 0^{\circ}-I 8^{\circ}$, fixating binocularly and in plane of the stimulus, but at different eccentricites. In each condition the first bar is for subject $\mathrm{AC}$, the second for BS

on this task it was not possible to assess the effects of the operations within animals. Nor were there significant differences between the thresholds of splenial and collicular animals $\left(\mathrm{U}=6, \mathrm{~N}_{1}=3, \mathrm{~N}_{2}=5, p=0.78\right.$, 2-tailed). It could be argued that this is weak evidence for the ineffectiveness of collicular damage on the detection of an increase in intensity, because of the absence of a pre-operative comparison. For this reason the entire procedure was repeated in animals 1-3 immediately after the second operation to ablate the superior colliculi, i.e. before they were retested on the movement discrimination. Table 2 shows that animals 1 and 3 actually improved postoperatively, perhaps reflecting the large number of trials required to reach a stable threshold again. Animal No. 2, which had the smallest collicular lesion, reached the beginning of criterion within 300 trials, with a slight and insignificant rise in threshold. Moreover, there was no indication of any impairment even during the first post-operative testing sessions, unlike the effect shown in Fig. 3 for movement discrimination. Overall we were therefore unable to find evidence that the collicular lesions impaired the detection of intensity changes. However, it should be noted that for technical reasons the final luminance of the stimulus spot in the intensity discrimination was approximately $1 \log$ unit greater than the luminance used for movement discrimination. This may well have influenced the results, as shown below in tests with human observers.

\section{Tests with human observers}

a. Movement. The results of measuring the smallest discernable movement under various viewing conditions are shown in Fig. 6. As the spot was always viewed from a fixed distance of 28.5 or $57 \mathrm{~cm}$ the results are shown in terms of visual angle. Under the best viewing conditions, i.e. head fixed and fixating binocularly in conditions $\mathrm{A}, \mathrm{C}$ and $\mathrm{I}$, the threshold was just below 2 min of arc. If the best monkey had been viewing from $28.5 \mathrm{~cm}$ its angular threshold would be $2.76 \mathrm{~min}$ of arc. For the two human observers the results of viewing at 28.5 and $57 \mathrm{~cm}$ were so similar that the results of these conditions were combined, i.e. $\mathrm{A}+\mathrm{C}$ and $\mathrm{B}+\mathrm{D}$.

Figure 6 shows that the threshold is raised by about $50-75 \%$ when the observer looks binocularly at infinity with the resulting blurred target on one fovea (conditions $\mathrm{B}$ and $\mathrm{D}$ ), when fixating the target monocularly (condition $G$ ), or when fixating infinity monocularly with the blurred target on the fovea (condition $\mathrm{H}$ ). Fixating binocularly but eccentrically in the plane of the target similarly raises the threshold (condition I). However, the most conspicuous elevation occurred in condition $F$, where the observer viewed binocularly but gazed at infinity and made no attempt, by keeping the head and eyes in one position, to ensure that the target was on either fovea. In this condition, which most closely resembles the viewing conditions of an unrestrained monkey that is unable to make appropriate vergence and fixation movements, the threshold was more than doubled.

b. Luminance increment. Figure 7 shows the mean intensity increment, expressed as a percentage of the final intensity of the spot (which was constant), for 3 human observers when fixating the spot or fixating eccentrically by up to $4^{\circ}$. When fixating the spot, the threshold varied from 2.4 to $4 \%$, which is substantially better than the lowest value of $10.5 \%$ shown by any monkey. However, eccentric fixation produced a wholly unforseen result. At an eccentricity of $1^{\circ}$, two observers improved substantially and one was unaffected. At $2^{\circ}$ one observer was still better than when fixating the spot and the other two were little worse. Only at an eccentricity of $4^{\circ}$ were all 3 observers worse. There is a ready explanation for these changes. In order to produce reliable changes in 


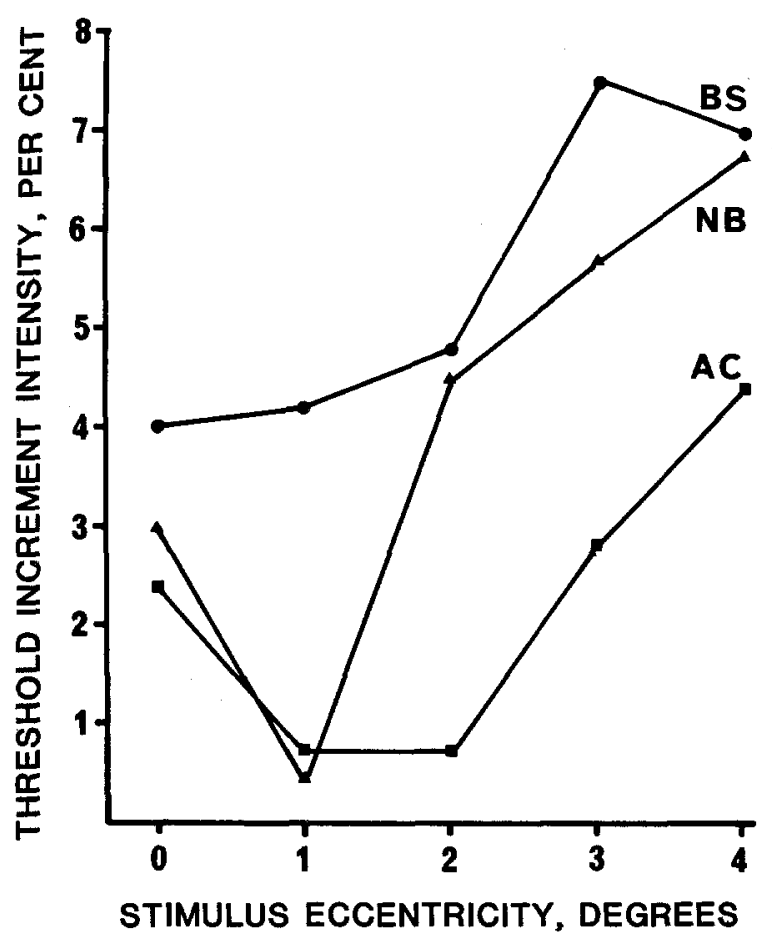

Fig. 7. Threshold increment intensity when fixating the luminous spot, or when fixating at various eccentricities to the right. There were 400 trials at each eccentricity for each observer. As the final intensity was constant the threshold increment is therefore given as a percentage of this value rather than the initial value. As the final intensity was $100 \mathrm{~cd} / \mathrm{m}^{-2}$ the ordinate can be read in these units as well as a percentage

intensity by varying the $Z$-axis voltage the intensity of the spot had to be increased almost tenfold from its value in the movement discrimination. This produced a faint but clear annulus about $1 \mathrm{~cm}$ in diameter around the spot, and with eccentric fixation of 0.5 and $1.0 \mathrm{~cm}$ the observer could readily detect the change in the annulus with the fovea. In other words, the effective stimulus was not eccentric at all. It was for this reason that we abandoned attempts to measure the increment threshold when fixating infinity because the spot or its annulus invariably lay within a few degrees of one or the other fovea.

\section{Discussion}

The primary purpose of the experiment was to investigate the effects of ablating the superior colliculi on the animals' ability to detect instantaneous movement of a single spot of light. The threshold elevation, which appeared to be permanent, was even greater than reported in an earlier investigation (Collin and Cowey 1980) in which two widely separated spots were presented and the animal had to detect which of them moved. We therefore conclude that the deficit in movement discrimination reported earlier can not be a consequence of selectively neglecting the far peripheral visual field, in which at least one of the two stimuli always had to fall. Nor can it result from an intensified saccadic suppression in a testing situation where the animal frequently changes its gaze from one stimulus to the other, as occurred in our earlier experiment. In the present experiment, where only one stimulus was present, all animals faced the display screen and with the exception of No. 3 they made very few head movements after starting a trial, presumably ensuring that the stimulus was always near the central retina. By the same reasoning the result also suggests that the demonstration by Anderson and Symmes (1969) that monkeys with collicular ablation were impaired at discriminating between two widely separated lines rotating at different rates, may not be simply explained by a peripheral neglect that makes it difficult for the animal to compare the fixated stimulus with the eccentric stimulus.

An alternative explanation for the impairment in movement discrimination, and for the apparent sparing of the discrimination of intensity (this experiment) and of pattern, flicker and colour (Anderson and Symmes 1969) is that cells in the mid-brain "are particularly sensitive to moving stimuli - as proposed by the latter authors and many others. This presumably implies that the cells in the mid-brain are even more sensitive to movement than cells in other visual areas, yet innumerable investigations show that the majority of cells in cortical visual areas are also more sensitive to moving than to stationary stimuli. These cortical visual cells hugely outnumber collicular cells. Why should they fail to detect visual movement when the colliculus is damaged? Whatever the nature of the visual neglect that follows midbrain damage perhaps it is not really restricted to or chiefly concerned with movement. Thus, in the experiment by Anderson and Symmes rate of movement was assessed by simultaneous visual discrimination between two widely separated targets, whereas colour, pattern and flicker were assessed by presenting a single stimulus on a central key. In other words the scanning requirements were different for impaired and unimpaired discriminations. More direct evidence that the superior colliculi and pretectum are involved in scanning is provided by the effects of damaging them on eye movements (Schiller et al. 1980) and on response latencies (Latto 1978; 
Collin et al. 1982) in visual search tasks. Furthermore, monkeys with mid-brain lesions are impaired at detecting brief flashes of light (Latto and Cowey 1971), fail to be distracted by flashed stimuli while performing a visual discrimination task (Milner et al. 1978) and are impaired at certain colour discriminations (Butter et al. 1982). In all these tasks the stimuli were stationary.

It should be pointed out that the absence of an impairment on the intensity task, a result compatible with imprecise fixation, could not arise simply as a consequence of the intensity task being easier than the movement task. In both tasks the same titration procedure stabilized performance at $79 \%$ correct. Nevertheless, the fact that the stimulus was much dimmer in the movement discrimination may have made it more difficult for animals with attentional disorders to detect and fixate the spot when it appeared.

Although there is no reason to suppose that oculomotor disorders are the prime cause of the sensory deficits that follow mid-brain damage, they may contribute to the disorder of movement perception, perhaps to exacerbate it and make it appear that movement is selectively impaired. Since monkeys with tectal and pretectal damage often give the impression of staring fixedly and slightly off-target and at a distant point we decided to investigate the effects on our own movement thresholds of relaxing accomodation and gazing at infinity. As Fig. 6F shows the threshold was doubled in one observer and tripled in the other when viewing in this way. Smaller elevations occurred with eccentric fixation in the plane of the stimulus, and when the stimulus, whether blurred or sharp, fell on the fovea of one eye only. Without objective measures of vergence, fixation, and accomodation it is not possible to be certain which of these factors may have contributed to the threshold elevation in monkeys. However, the effect on monkeys of physically blurring the stimulus (Fig. 4) or temporarily relaxing accommodation (Fig. 5) indicates that image blurring alone has too slight an effect on the movement threshold to fully account for the defects. This is confirmed by comparing thresholds in human observers when the stimulus is fixated monocularly (Fig. 6G) or when the viewing eye is accommodated for infinity but the stimulus lies on the visual axis (Fig. $6 \mathrm{H}$ ). Blurring the image in this way had no effect in one observer and only a small effect in the other. Imprecise fixation seems a more likely candidate, for when human observers fixated eccentrically but with appropriate accommodation for the stimulus there was a roughly linear relation between threshold and eccentricity. But even with $8^{\circ}$ eccentricity the threshold was barely doubled. This certainly resembles the final effect of mid-brain lesions but falls well short of the initial effects. Furthermore, monkeys with collicular ablation can fixate accurately when required to do so in order to present other stimuli or to obtain a reward (Wurtz and Goldberg 1972; Schiller et al. 1980; Mohler and Wurtz 1977; Albano and Wurtz 1982). Of course, there was no requirement that our monkeys should fixate accurately, nor did they have more than two seconds in which to do so on each trial. In addition, the caudal pretectum was damaged in our animals and pretectal damage does permanently impair saccades, and accurate fixation of targets that are not in the straight-ahead position (Albano and Wurtz 1982). But the inaccurate fixation is unlikely to be extensive because the animals were not impaired on the increment intensity task despite the fact that human observers were impaired when fixating eccentrically by $3-4^{\circ}$, although not by $1-2^{\circ}$ (Fig. 7). The latter result is especially interesting because it shows that small eccentric fixations that can elevate movement thresholds for a dim stimulus have no effect or even reduce the threshold when detecting an increase in the intensity of a brighter but otherwise similar target. It may partly explain why the monkeys were impaired at detecting movement but not changes in intensity, without supposing any special role for the colliculus in the detection of movement. Of course, this interpretation points to the need to measure eye movements in further investigations of the effects of collicular damage on sensory thresholds.

A further piece of evidence which supports the hypothesis that the animals may not have fixated in the correct plane stems from the effects of similar mid-brain ablations on stereopsis and pattern perception (Lawler 1981; Cowey 1984). Lawler found that combined tectal and pretectal damage abolished or greatly impaired global stereopsis for stereograms presented close to the animal. Additionally, she found that similarly close vertical line targets first presented monocularly to the monkeys were treated by the animals as if the number of lines had doubled when they were subsequently seen binocularly, i.e. as if the animals had horizontal diplopia. Both the impaired global stereopsis and the diplopia are consistent with convergence paresis and could readily be demonstrated in human observers by asking them to look at infinity while inspecting targets very close to them.

The nystagmus produced by bodily rotation in the dark is suppressed if a visible surround is also rotated, either with monkeys (Waespe and Henn 1978) or human subjects (Waespe et al. 1980). Nystagmus induced by caloric stimulation of the 
vestibular system is similarly suppressed in the light. Similarly, unilateral lesions of the paramedian pontine reticular formation induce a spontaneous nystagmus directed contralaterally in the dark and which is suppressed in the light (Cohen et al. 1968). If the monkeys with pre-tectal damage had spontaneous nystagmus in the dark, and the solitary luminous stimulus was insufficient to suppress it, the movement threshold would presumably be impaired. Future experiments will examine this, but if nystagmus were present it is difficult to see why the intensity threshold was not also impaired.

The above discussion shows that there is evidence from a variety of sources that part of the impairment in movement discrimination following mid-brain damage may be a secondary consequence of an oculomotor disorder rather than a defect in the physiological mechanisms for detecting movement itself. It is equally important to consider which midbrain regions are most involved in the disorder. When the damage spared the rostral superior colliculi (animal 2) there was no impairment. Although this could indicate that the rostral colliculus is important for the perception of movement in foveal vision (which is represented in rostral colliculus) and/or for normal eye movements, this was also the only animal in which the pretectum was almost entirely spared. In much earlier experiments in which almost the entire superior colliculi were ablated with little involvement of the pre-tectum it was reported that eye movements were normal, whereas saccades and even vergence were grossly abnormal following selective pretectal damage (Pasik et al. 1966, 1969a, b; Pasik and Pasik 1975). If the damage involved the posterior commissure directly, or indirectly by destruction of the nucleus interstitialis, vertical eye movements were grossly impaired (see Bender 1980, for review). In the present experiment, the extent to which eye movements were abnormal, the eyelids were retracted, and the pupils slightly expanded all correlated with the extent of pre-tectal damage. The correlation between pre-tectal damage and elevation of movement threshold was equally clear. Animal 2 was unimpaired and had no pre-tectal damage. Animals 1 and 4 had small impairments, only slight pre-tectal damage, and either no or slight demyelination in the posterior commissure. Animals 5-8, which had the largest discrimination impairment, all had extensive damage to the caudal pre-tectum in a position that could interrupt fibres passing from nucleus interstitialis of the posterior commissure (just beneath the olivary nucleus and the remainder of the caudal pretectum) to the posterior commissure itself. It is therefore not surprising that the sections stained for fibres showed extensive demyelination of the posterior commissure in these 4 animals. We therefore conclude that pretectal rather than tectal damage is responsible for the oculomotor disorders and the resulting disturbance in detecting movement of a small luminous target. Further localisation may not be justified by the present evidence but the caudal pretectum involving the olivary nucleus, nucleus interstitialis and (indirectly) the posterior commissure seems to be essential for normal oculomotor behaviour and detection of fine movement. It must also be remembered that there are reciprocal connexions between the frontal eye-fields and the pre-tectum (Leichnetz 1982a) and that deep pre-tectal lesions may interrupt the prefrontal oculomotor bundle (Leichnetz 1982b) en route to the rostrolateral oculomotor nucleus.

Finally, our results can be briefly related to pathological conditions in man. Although oculomotor disorders have been related to damage in widespread areas of the cortex and sub-cortex (partly because the degenerative lesions are often diffuse) there is clear clinical evidence that parts of the brain stem are particularly important (see Hoyt and Daroff 1971, for a review of the voluminous literature). Imprecise fixation was described by Holmes (1936, 1938) following damage centred on the brachium of the superior colliculus, which of course contains the pre-tectal fibres, and the adjacent medial pulvinar. Poor fixation and vergence as a consequence of inadequate and infrequent saccadic and disjunctive eye movements, first reported by Parinaud (1886), is also a feature of supra-nuclear palsy (Steele et al. 1964). The pathological changes in this disease are commonest in the mid-brain reticular formation, zona incerta and central grey but also include the ventro-lateral pretectal region bordering the tegmentum. Unilateral vascular pretectal lesions were associated with vertical paresis of one eye, with accompanying vertical diplopia, by Jampel and Fells (1968), and dorsal mid-brain stereotaxic lesions in man at the level of the posterior commissure produced a persisting paralysis of vertical eye movements (Nashold and Gills 1967). If the highly circumscribed lesions of the dorsal mid-brain in our animals produced similar, albeit less extreme; symp-... toms it is no longer difficult to see why prompt and accurate fixation are difficult and why movement thresholds are elevated. There certainly seems no need to postulate the removal of a specialized set of movement detectors to explain the consequences of tectal and pre-tectal lesions; rather, the present findings can be understood in terms of a general sensory deficit in which small movements are especially difficult to detect because they require accurate fixation. 
Acknowledgements. This research was supported by MRC grant G971/397/B. We thank C. Pyrah for histological help and Dr. S. Judge for many helpful suggestions and comments on the manuscript.

\section{References}

Albano JE, Mishkin M, Westbrook LE, Wurtz RH (1982) Visuomotor deficits following ablation of monkey superior colliculus. J Neurophysiol 4: 338-351

Albano JE, Wurtz RH (1982) Deficits in eye position following ablation of monkey superior colliculus, pretectum and posterior medial-thalamus. J Neurophysiol 48: 318-337

Anderson KV, Symmes D (1969) The superior colliculus and higher visual functions in the monkey. Brain Res 13: 37-52

Bender MB (1980) Brain control of conjugate and vertical eye movements. A survey of the structural and functional correlates. Brain 103: 26-69

Butter CM, Kurtz D, Leiby CC, Campbell A (1982) Contrasting behavioral methods in the analysis of vision in monkeys with lesions of the striate cortex or the superior colliculus. In: Ingle DJ, Goodale MA, Mansfield RJW (eds) Analysis of Visual Behavior. MIT Press, Cambridge, pp 301-334

Cohen B, Komatsuzaki A, Bender MB (1968) Electro-oculographic syndrome in monkeys after pontine reticular formation lesions. Arch Neurol Chicago 18: 78-92

Collin NG, Cowey A (1980) The effect of ablation of frontal eyefields and superior colliculi on visual stability and movement discrimination in rhesus monkeys. Exp Brain Res 40: 251-260

Collin NG, Cowey A, Latto R and Marzi C (1982) The role of frontal eye-fields and superior colliculi in visual search and non-visual search in rhesus monkeys. Behav Brain Res 4: 177-193

Cowey A (1984) Disturbances of stereopsis by brain damage. In: Ingle D (ed) Brain Mechanisms and Spatial Vision. Nato Animal Study Institute Series. Martinus Nijholf (in press)

Cynader M, Berman N (1972) Receptive-field organization of monkey superior colliculus. J Neurophysiol 35: 187-201

Denny-Brown D (1962) The midbrain and motor integration. Proc R Soc Med 55: 527-538

Goldberg ME, Wurtz RH (1972) Activity of superior colliculus in behaving monkey. I Visual receptive fields of single neurons. J Neurophysiol 35: 542-549

Holmes G (1936) Looking and seeing. (Movements and fixation of the eyes.) Irish J Med Sci 129: 565-576

Holmes G (1938) The cerebral integration of the ocular movements. Br Med J 2: 107-112

Horn G, Hill RM (1966) Responsiveness to sensory stimulation of units in the superior colliculus and subjacent tectotegmental regions of the rabbit. Exp Neurol 14: 199-223

Hoyt WF, Daroff RB (1971) Supranuclear disorders of ocular control systems in man. In: Bach-y-Rita P, Collins CC, Hyde JE (eds) The Control of Eye Movements. Academic Press, New York, pp 175-235

Humphrey NK (1968) Responses to visual stimuli of units in the superior colliculus of rats and monkeys. Exp Neurol 20: 312-340

Jampel RS, Fells P (1968) Monocular elevation paresis caused by a central nervous system lesion. Arch Ophthalmol 80: 45-57

Latto RM (1978) The effects of bilateral frontal eye-field, posterior parietal or superior collicular lesions on visual search in the rhesus monkey. Brain Res 146: 35-50
Latto R, Cowey AC (1971) Visual field defects after frontal eyefield lesions in monkeys. Brain Res 30: 1-24

Leichnetz Gr (1982a) Connections between the frontal eye field and pretectum in the monkey: An anterograde/retrograde study using HRP gel and TMB neurohistochemistry. J Comp Neurol 207: 394-402

Leichnetz GR (1982b) The medial accessory nucleus of Bechterew: A cell group within the anatomical limits of the rostral oculomotor complex receives a direct prefrontal projection in the monkey. J Comp Neurol 210: 147-151

Marroco RT, Li RH (1977) Monkey superior colliculus: properties of single cells and their afferent inputs. J Neurophysiol 40: 844-860

Milner AD, Foreman NP, Goodall MA (1978) Go-left go-right discrimination performance and distractibility following lesions of prefrontal cortex or superior colliculus in stumptail macaques. Neuropsychologia 16: 381-390

Mohler CW, Wurtz RH (1977) Role of striate cortex and superior colliculus in visual guidance of saccadic eye movements in monkeys. J Neurophysiol 40, 74-79

Nashold BS, Gills JP (1967) Ocular signs from brain stimulation and lesions. Arch Ophthalmol 77: 609-618

Parinaud H (1886) Paralysis of the movement of convergence of the eyes. Brain 9: 330-341

Pasik T, Pasik P, Bender MB (1966) The superior colliculi and eye movements. Arch Neurol 15: 420-436

Pasik P, Pasik T, Bender MB (1969a) The pretectal syndrome in monkeys. I. Disturbances of gaze and body posture. Brain 92: 521-534

Pasik T, Pasik P, Bender MB (1969b) The pretectal syndrome in monkeys. II. Spontaneous and induceds nystamies and "lightning" eye movements. Brain 92: 871-884

Pasik T, Pasik P (1975) Experimental models of oculomotor dysfunction in the rhesus monkeys. In: Meldrum BS, Marsden CD (eds) Advances in Neurology, Vol 10. Raven Press, New York, p 77-89

Robinson DL, Wurtz RHI (1976) Use of an extraretinal signal by monkey superior colliculus neurons to distinguish real from self-induced stimulus movements. J Neurophysiol 39: 852-870

Schiller PH, Koerner F (1971) Discharge characteristics of single units in superior colliculus of the alert rhesus monkey. $\mathrm{J}$ Neurophysiol 34: 920-936

Schiller PH, True SD, Conway JL (1980) Deficits in eye movements following frontal eye-field and superior colliculus ablations. J Neurophysiol 44: 1175-1189

Steele JC, Richardson JC, Olszewski J (1964) Progressive supranuclear palsy. Arch Neurol 10: 333-359

Waespe W, Henn V (1978) Conflicting visual-vestibular stimulation and vestibular nucleus activity in alert monkeys. Exp Brain Res 33: 203-211

Waespe B, Waespe W, Henn V (1980) Subjective velocity estimation during conflicting visual-vestibular stimulation. Arch Psychiat Nervenkr 228: 109-116

Wetherill GB, Levitt H (1965) Sequential estimation of points on a psychometric function. Br J Math Statist Psychol 50: 1=10

Wurtz RH, Goldberg ME (1972) Activity of superior colliculus in behaving monkey. IV. Effects of lesions on eye movements. J Neurophysiol 35: 587-596

Received November 4, 1983 / Accepted March 6, 1984 